\title{
The role of eye movements in the Müller-Lyer illusion
}

The size of the illusion was found to be unaffected by reducing the exposure of the figures tachistoscopically, or by reducing the size of the figures. The illusion therefore does not seem to be due to eye movements or a tendency to make eye movements.

One of the oldest and best known hypotheses to account for the Müller-Lyer illusion is Külpe's proposal that it is due to eye movements. He proposed that the tail of the arrow pulls the eye out from the shaft while the head of the arrow pulls the eye in, and that eye movement along the shaft rather than the objective length of the shaft is the basis of the judgment of length. This was an attractive hypothesis, and it was supported by early observations of eye movements (e.g., Judd, 1905). Judd's Ss did make more extensive saccadic movements while observing the double tail than while observing the double head.

There remained some question, however, whether the perceptual illusion is due to eye movement, or whether both the illusion and the eye movements are the result of some more basic perceptual mechanism. At this point Lewis (1908) found that the illusion was undiminished with tachistoscopic presentation of the figures, and suggested that rather than being due to actual eye movements, it might be attributed to a "tendency" to make eye movements.

The intervening years have produced no completely adequate explanation of the illusion, and both the original actual eye movement hypothesis and the modified "tendency" hypothesis are still invoked. Thus, Festinger, White, and Allyn (1968) attribute the illusion to what they call efferent readiness. The illusion occurs, they say, because of a propensity to move the eyes, regardless of whether such movements actually occur, or are precluded by rapid exposure (Lewis, 1908; Yarbus, 1967) or by the use of a stabilized image technique (Yarbus, 1967).

Previous investigators of the Müller-Lyer illusion have typically used figures that subtended a visual angle of $5 \mathrm{deg}$ or $6 \mathrm{deg}$, and in such an instance eye movement is necessary to bring the whole figure into foveal vision. In the absence of any clear statement to the contrary, it may be assumed that it is the resulting movement to fixate to which the eye-movement theorist refers. This implication was tested in the present study by presenting figures that were reduced in size so that they fell entirely on the fovea, thus precluding the necessity for eye movements and even the "tendency" to make eye movements. A second objective was simply to confirm the finding of earlier investigators that the illusion is not diminished by brief figure presentation. For that purpose, both normal-sized and reduced figures were presented tachistoscopically.

\section{METHOD}

The Ss were 10 introductory psychology students.

The size of the Müller-Lyer illusion was determined with the method of constant stimuli. The shaft of the double tail was $5 \mathrm{~cm}$ long while the shaft of the double head was $4.5,5.0,5.5,6.0$, and $6.5 \mathrm{~cm}$ on different stimulus figures. The former was always parallel to and centered $3 \mathrm{~cm}$ above the latter. All lines were approximately $.7 \mathrm{~mm}$ thick. The angles of the head and tail were $1 \mathrm{~cm}$ long and formed an angle of $22.5 \mathrm{deg}$ with the shaft. The
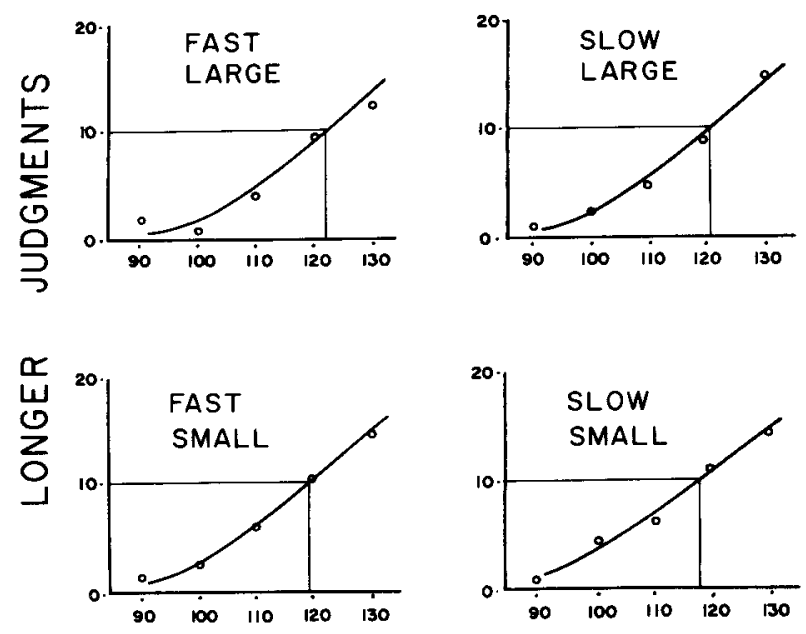

\section{PERCENTAGE OF STANDARD STIMULUS}

Fig. 1. Judgments of longer for the double head as function of its length relative to the double tail.

stimulus figures were glossy photographic reproductions either full-size or reduced to $1 / 10$ size. They were presented centered about the fixation point of an illuminated field, at a viewing distance of approximately $60 \mathrm{~cm}$ so that the large figures presented a visual angle of $5 \mathrm{deg}$ and the small ones a visual angle of $0.5 \mathrm{deg}$.

The Ss were given some preliminary training with the tachistoscope judging the sizes of rectangles, and then were given four series of Müler-Lyer figures. The series were presented under the following conditions: large figures at a .05-sec exposure, large figures at a 1-sec exposure, small figures at $.25 \mathrm{sec}$ (it had been previously found that the small figures could not be adequately seen at exposures much shorter than $.25 \mathrm{sec}$ ), and small figures at $1 \mathrm{sec}$. A ready signal preceded each presentation. Within each series each figure was presented twice in a random order. Ss were asked to judge whether the horizontal line in the double head was longer than, equal to, or shorter than the one in the double tail.

\section{RESULTS AND DISCUSSION}

The results for the four sets of presentation conditions are shown in Fig. 1. The ordinate gives the total number of judgments of longer out of a possible 20 (10 Ss judging each pair of stimuli twice). Equal judgments were scored as $1 / 2$ point for longer. The abscissa gives the relative lengths of the variable (double head) and standard (double tail) stimuli.

In spite of the relatively small number of observations, the psychometric functions are quite orderly, and in all cases appear to justify interpolation to determine the point of subjective equality, and hence an estimate of the magnitude of the illusion under the four conditions. These magnitudes appear to cluster 
closely together; the illusion constitutes very nearly $20 \%$ of the length of the standard stimulus regardless of whether the figures were full-sized or reduced, or whether they were presented for 1 sec or for a much shorter time.

It is evident that reducing stimulus exposure to $.05 \mathrm{sec}$ fails to eliminate the illusion, so that we may conclude, as Lewis did, that the illusion does not require the opportunity for making eye movements.

That the illusion is not diminished when the figure subtends a visual angle of only $0.5 \mathrm{deg}$, so that it falls entirely on the fovea, would seem to argue against the idea that the illusion is due to a "tendency to make" or a "motive for" or an "efferent readiness of" eye movements. There are, to be sure, minute eye movements, tremor, that cover fields this small (Ratliff \& Riggs, 1950), but it is hard to believe after Yarbus's (1967) results that these are the movements that have been assumed to produce the illusion. And if, as the present results show, needing or not needing to make fixation movements, saccades, makes no difference in the magnitude of the illusion, then it is not clear just what kind of eye movements the illusion is supposed to depend upon or how one could determine what role they play in producing it. It now appears much more likely that the illusion is a result of central or peripheral sensory mechanisms that create both the distorted perception and the tendency to make differential eye movements. Hopefully, continued study of the illusion will help clarify how these sensory mechanisms work.

\section{REFERENCES}

FESTINGER, L., WHITE, C. W., \& ALLYN, M. R. Eye movements and decrements in the Muller-Lyer illusion. Perception \& Psychophysics, $1968,3,376-382$.

JUDD, C. H. The Müller-Lyer illusion. Psychological Review Monographic Supplement, 1905, 7, 55-81 (Whole No. 29).

LEWIS, E. O. The effect of practice on the perception of the Muller-Lyer illusion. British Journal of Psychology, 1908, 2, 294-306.

RATLIFF, F., \& RIGGS, L. A. Involuntary motions of the eye during monocular fixation. Journal of Experimental Psychology, 1950, 40, 687.701.

YARBUS, A. L. Eye movements and vision. Translated by B. Haigh. New York: Plenum Press, 1967.

\section{NOTE}

1. Address: Department of Psychology, University of Washington, Seattle, Washington 98105.

(Accepted for publication February 3, 1969.) 